\title{
The NIST Optically Pumped Cesium Frequency Standard
}

\author{
Robert E. Drullinger, David J. Glaze, J. L. Lowe, and Jon H. Shirley
}

\begin{abstract}
The National Institute of Standards and Technology is developing an optically pumped, cesium-beam primary frequency standard. The design follows from an analysis of systematic errors found in cesium beam standards. The details of the design, performance of major subsystems, and preliminary tests of the standard are presented.
\end{abstract}

\section{INTRODUCTION}

$\mathrm{W}$ E have previously experimented with optical state preparation and detection in small prototype cesium beam tubes [1]. These experiments indicated that we could construct a large, primary standard which would have exceptional shortterm stability and essentially eliminate several of the accuracylimiting systematic errors found in conventional cesium standards.

A project was begun to build a large prototype optically pumped standard capable of continuous clock operation and semiautomatic self-evaluation. However, to achieve the full accuracy potential, careful consideration had to be paid to all sources of systematic error. These studies are outlined in the section on systematic effects.

Following this analysis, the design of the beam tube is outlined in the next section. The laser technology necessary to realize the performance potential is briefly outlined in the section on lasers and the requirements on the control electronics are outlined in the following section. In the final section some preliminary results are presented.

\section{Systematic Effects}

The systematic effects which have been analyzed or re-analyzed include: fluorescent light shift [2]; velocity-dependent effects such as second-order Doppler shift and end-to-end cavity phase shift, including their dependence on RF power and modulation parameters; Rabi-pulling; cavity pulling; Majorana effects; distributed-cavity phase shift [3]; RF spectral purity; and magnetic field uniformity.

Many of the shifts can be expressed as the ratio of two integrals over the velocity distribution containing factors dependent on the microwave power, the modulation parameters, and the particular shift mechanism. In the specific case of second-order Doppler shift in the limit of slow modulation rate:

$$
\frac{\Delta \nu_{D}}{\nu_{0}}=-\frac{L^{2}}{2 c^{2} T_{D}^{2}}
$$

Manuscript received June 12, 1990; revised August 27, 1990.

The authors are with the Time and Frequency Division, National Institute of Standards and Technology (NIST), Boulder, CO 80303

IEEE Log Number 9041638. where $L$ is the length of the drift region, $c$ is the speed of light and the effective transit time $\left(T_{D}\right)$ is given by the ratio

$$
T_{D}^{2}=\frac{\left\langle T^{2} M\left(\omega_{m} T\right) \sin ^{2} 2 b \tau\right\rangle}{\left\langle M\left(\omega_{m} T\right) \sin ^{2} 2 b \tau\right\rangle}
$$

The angle brackets denote an average over the atomic velocity distribution. The power dependence of the shift enters through the factor $\sin ^{2} 2 b \tau$. The modulation dependence enters through the factor $M\left(\omega_{m} T\right)$ where $\omega_{m}$ is the modulation amplitude. In the absence of modulation $M=1$. For slow sine wave frequency modulation $M(x)=(2 / x) J_{1}(x)$. For slow square wave modulation $M(x)=(1 / x) \sin x$. At higher modulation rates the $M$ 's become more complicated and also depend on the modulation frequency. For very narrow velocity distributions, the velocity average can be ignored, and the power and modulation dependent factors cancel. The shifts then have little or no dependence on microwave power or modulation parameters. An optically pumped standard, however, will use almost all of the broad thermal distribution of velocities emerging from the oven. The shifts then acquire significant dependence on microwave power and modulation parameters. For example, the secondorder Doppler shift and end-to-end cavity phase shift can change by 5 to $10 \%$ with microwave power changes of only $1 \mathrm{~dB}$.

Second-order Doppler shifts may be calculated if the effective velocity profile, effective microwave power, and modulation parameters are known to adequate accuracy. However, to predict the necessary information from operational parameters seems risky at best. We have developed a numerical method for extracting both the velocity distribution and the effective microwave power level from Ramsey lineshapes [4]. Tests with theoretical lineshape data, even with substantial noise added, show that the extracted distributions are adequate to permit a $1 \%$ evaluation of the second-order Doppler shift.

Rabi-pulling and Majorana effects should be extremely small in this standard but the theoretical studies give new insight into how these effects enter a standard. These studies are briefly outlined in [5] and more detailed publications are in preparation.

\section{ATOMic BEAM Tube}

The geometry of the new beam tube is outlined in Fig. 1. The device is totally symmetric about the central microwave feed point so only half the tube is shown. The design logic and major subsystems have been described previously [6]. The Ramsey cavity ends are a new concept, designed to minimize distributed-cavity phase-shift [3]. An axial quantization field has been chosen to minimize Rabi-pulling [7] and to provide better magnetic field uniformity. The fluorescence collection optics are large-radius spherical mirrors, which collect $50 \%$ of the fluorescent light and inject it into a light guide for detection out- 


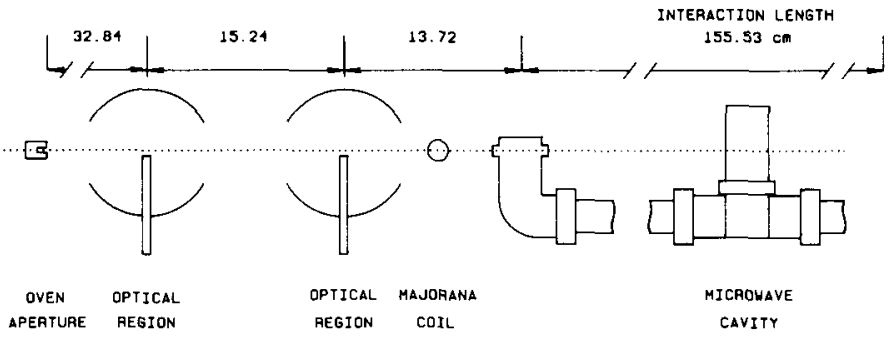

Fig. 1. Schematic diagram of one end of the beam tube (dimensions are in centimeters).

side of the vacuum envelope. The imaging nature of this system provides the necessary high selectivity against scattered light. All laser optics are external to the beam tube. The laser beams enter and exit through laser quality, normal incidence, antireflection-coated windows.

The $\mathrm{C}$-field solenoid extends over the entire length of the standard. Three layers of passive magnetic shielding provide a static, axial shielding factor of $6 \times 10^{3}$. This will be electronically enhanced by servo control of the C-field.

The atomic beam is $3 \mathrm{~mm}$ in diameter and is derived from a new oven design with a recirculating collimator [8]. This oven, when operated at $110^{\circ} \mathrm{C}$, produces a flux of $5 \times 10^{9}$ atoms $/ \mathrm{s}$ at the detector while its total cesium emission is only 30 $\mathrm{mg} /$ year. This atomic flux can be combined with the geometry of the standard to predict the short-term stability to be expected [9], [10]. When the standard is operated with a single laser for state preparation, i.e., no $m_{F}$ substate pumping, and detection via a cycling transition, one expects $\sigma_{y}(\tau) \approx 3.5 \times$ $10^{-13} / \sqrt{\tau}$.

The vacuum chamber is $25 \mathrm{~cm}$ in diameter and $2.5 \mathrm{~m}$ long. It has all-metal seals with the exception of small elastomer seals around the light pipes and on the oven valves. Also, the laser windows are attached with a special low-strain vacuum epoxy. The system is pumped with two ion pumps with a total pumping speed of $601 / \mathrm{s}$. Two of the magnetic shields are inside the vacuum system and the third is outside. The chamber is temperature regulated by the addition of a nonmagnetic heater directly on the surface of the vacuum tube. The space $(5 \mathrm{~cm})$ between the chamber and the outer shield is filled with thermal insulation. This heater/oven assembly is used both for vacuum bake-out and for thermostatic control of the temperature of the chamber during operation.

\section{LASER SYSTEMS}

It has been shown that simple, "off-the-shelf" laser diodes with their inherent FM noise and resulting linewidths of many megahertz are incapable of supporting optically pumped clock operation at full atomic-shot-noise-limited performance [1]. To solve this problem we have developed a laser-line-narrowing technique based on optical feedback from a high-Q cavity [11] (see Fig. 2). With this line-narrowing technique, we have demonstrated essentially atomic-shot-noise-limited performance in an optically pumped standard [12].

\section{Control Electronics}

The design of the beam tube produces a clock-signal line $Q$ of just over $10^{8}$. When combined with the design goal of $10^{-14}$ for frequency accuracy, the line-center accuracy requirement on the main frequency control servo becomes something better

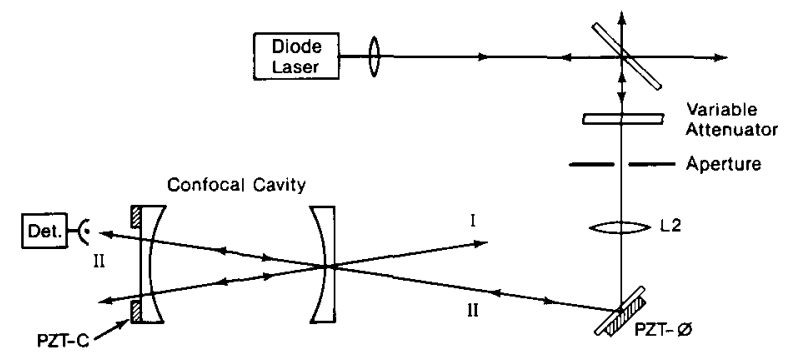

Fig. 2. Scheme for laser line narrowing by optical feedback from a cavity

than a part in $10^{6}$. At the time the project was begun it was felt that available crystal technology would not support a slow square-wave modulation scheme because of noise in the crystal at the second harmonic of the modulation frequency [13]. However, to achieve this accuracy in a traditional, linear (sine-wavephase-modulation) servo required the development of a new modulation technique with second harmonic distortion below $-120 \mathrm{~dB}$. This servo (to be described in a subsequent publication) will be used in our initial operation of the new standard.

As pointed out in the section on systematic effects, optically pumped standards are relatively sensitive to changes in microwave power. Furthermore, our choice of an axial C-field combined with the reduced axial shielding effect of long cylindrical shields produces an undesirable sensitivity to the magnetic environment. To solve these problems, we would like to develop signals directly from the atomic spectrum and to servo control both the applied microwave power and the effective $\mathrm{C}$-field value. To accomplish this and to provide the self-evaluation capabilities inherently feasible in this standard, it is desirable to use a digital servo control system. Such a system would most logically use a slow square-wave modulation scheme. Recently developed crystal technology will support such a servo [14] and we plan to acquire or develop a second generation of electronics that incorporates these features.

\section{RESULTS}

The beam tube has been completed and preliminary tests have been run. With the chamber operated at $40^{\circ} \mathrm{C}$ the pressure is $\leq 2 \times 10^{-6} \mathrm{~Pa}$. The fluorescence-collection optics have been shown to collect $50 \%$ of the light; even in the preliminary work with less than optimum laser beam profiles, the signal is many times that of the scattered light. The $\mathrm{C}$-field was measured to be uniform to well within the system requirements and the spectrum of Zeeman-sensitive transitions confirms this. Electrical measurements made on the microwave cavity during assembly show an end-to-end asymmetry of $5 \mu \mathrm{m}$ and an asymmetry in the ends that should lead to a distributed-cavity phase shift of $\leq 3 \times 10^{-6} \mathrm{rad}$.

Preliminary operation of the beam tube has been performed with a single laser narrowed by optical feedback and locked to the $F=4 \rightarrow F^{\prime}=4$ transition in a separate saturated absorption cell. This laser was used to drive the pumping zone with an optical power density of about $5 \mathrm{~mW} / \mathrm{cm}^{2}$. The polarization was linear, but neither pure $\pi$ nor pure $\sigma$. Pure $\pi$ polarized light does not pump the $m=0$ substate, while $\sigma$-polarized light can produce a coherence trapping phenomenon [15]. However, with the low magnetic field and short optical interaction time employed, the magnetic field does not clearly define a quantization axis and coherence trapping may still have occurred. A second 

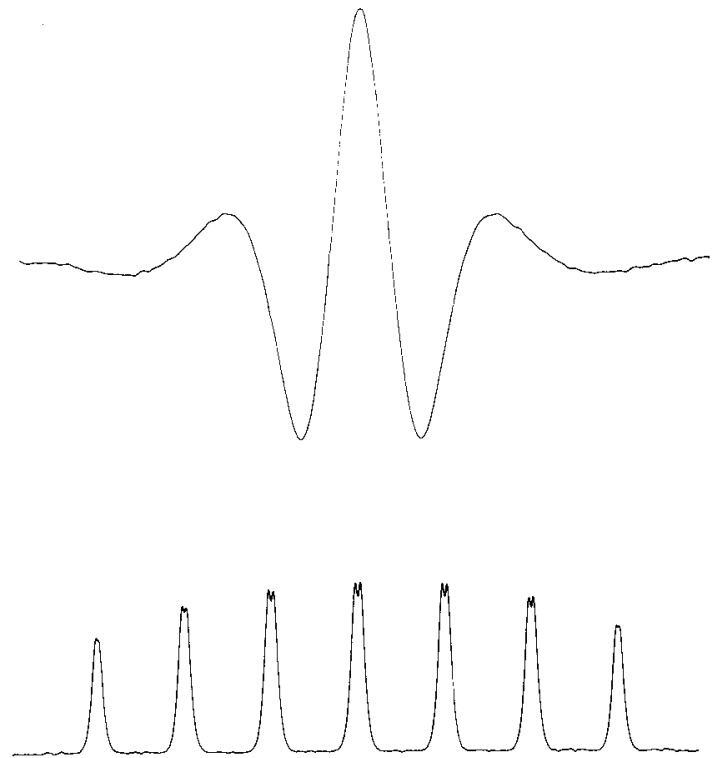

Fig. 3. Spectra from NIST-7. The upper trace is the central Ramsey feature at optimum power $(800-\mathrm{Hz}$ scan $)$. The lower trace is the entire Zeeman spectrum $(600-\mathrm{kHz}$ scan $)$

light beam was synthesized from the first with an acoustooptic device and used to drive the detection zone on the $F=4 \rightarrow F^{\prime}$ $=5$ cycling transition with a power density of about $1 \mathrm{~mW} / \mathrm{cm}^{2}$.

Fig. 3 shows a complete scan of the microwave spectrum as well as the Ramsey structure on the central peak. The scans were taken at optimum microwave power. The observed Ramsey line width of $65 \mathrm{~Hz}$ is in agreement with theoretical predictions based on the cavity length, oven temperature, and a thermal velocity distribution weighted by $1 / v$ [9]. The broad scan shows the Rabi line shapes expected for the chosen microwave excitation profile. The absence of $\Delta m= \pm 1$ transitions implies that the field orientation is good. The dip in the center of the Rabi peaks is a natural result of the microwave power level and the very broad velocity distribution. The Ramsey structure does not show on the broader scan because of scan speed and instrument response time.

\section{SUMMARY}

We have developed an optically pumped, cesium-beam, primary frequency standard. We plan to use this device to study the physics of ac stark shifts as well as Majorana effects. The device is designed to have a short-term stability characterized by $\sigma_{y}(\tau) \approx 3.5 \times 10^{-13} / \sqrt{\tau}$, an accuracy of one part in $10^{14}$, to run continuously as a clock and to be nearly self-evaluating. Although the standard has only operated in a very preliminary way, at this time there is no indication that the design goals will not be reached.

\section{ACKNOWLEDGMENT}

The authors would like to acknowledge the many people whose useful discussions and collaborations have affected the design of the new standard. In particular, we wish to recognize the contributions of Prof. Andrea DeMarchi and Dr. L. Cutler.

\section{REFERENCES}

[1] A. Derbyshire, R. E. Drullinger, M. Feldman, D. J. Glaze, D. Hillard, D. A. Howe, L. L. Lewis, J. H. Shirley, I. Pascaru, and D. Stanciulescu, "Optically pumped small cesium beam standards: A status report," in Proc. 39th Annu. Symp. Frequency Control, pp. 18-21, 1985.

[2] J. Shirley, "Fluorescent light shift in optically pumped cesium standards," in Proc. 39th Annu. Symp. Frequency Control, pp. $22-23,1985$

[3] A. DeMarchi, J. Shirley, D. J. Glaze, and R. Drullinger, "A new cavity configuration for cesium beam primary frequency standards," IEEE Trans. Instrum. Meas., vol. 37, pp. 185-190, June 1988.

[4] Jon H. Shirley, "Velocity distributions from Fourier transforms of Ramsey line shapes," in Proc. 43rd Annu. Symp. Frequency Control, pp. 162-167, 1989.

[5] R. E. Drullinger, J. H. Shirley, D. J. Glaze, and L. Hollberg, "An optically pumped primary frequency standard," in Proc. Frequency Standards and Metrology, A. DeMarchi, Ed. New York: Springer-Verlag, 1988, pp. 116-118.

[6] R. E. Drullinger, J. Shirley, D. J. Glaze, L. W. Hollberg, and A. DeMarchi, "Progress toward an optically pumped cesium beam frequency standard,"' in Proc. 40th Ann. Symp. Frequency Control, pp. 428-431, 1986.

[7] A. Demarchi, G. D. Rovera, and A. Premoli, "Pulling by neighbouring transitions and its effects on the performance of cesiumbeam frequency standards,"'Metrologia, vol. 20., pp. 37-47, 1984.

[8] R. E. Drullinger, D. J. Glaze, and D. B. Sullivan, "A recirculating oven for atomic beam frequency standards,"' in Proc. 39 th Annu. Symp. Frequency Control, pp. 13-17, 1985.

[9] C. Audoin, V. Candelier, and J. Vanier, "Effect of the atomic transit time on the frequency stability of cesium beam frequency standards," IEEE Trans. Ultrasonics, Ferroelectrics, Frequency Control, vol. 34, pp. 573-581, Nov. 1987.

[10] V. Giordano, V. Candelier, A. Hamel, C. Audoin, G. Théobald, and P. Cérez, "Noise in the optical detection of atoms in a beam," Optics Communications, vol. 67, no. 4, pp. 287-292, July 1988.

[11] B. Dahmani, L. Hollberg, and R. Drullinger, "Frequency stabilization of semiconductor lasers by resonant optical feedback,' Opt. Lett., vol. 12, pp. 876-878, Nov. 1987.

[12] S. Ohshima, Y. Koga, Y. Nakadan, L. Hollberg, and R. Drullinger, "The effect of laser line narrowing on the performance of optically pumped cesium atomic beam frequency standards," in Proc. 2nd European Frequency and Time Forum, pp. 531-536, 1988.

[13] F. L. Walls, "Stability of frequency locked loops," in Proc. Frequency Standards and Metrology, A. DeMarchi, Ed. New York: Springer-Verlag 1988, pp. 145-149.

[14] F. W. Walls, NIST, 325 Broadway, Boulder Colorado, private communication.

[15] G. Théobald, P. Cérez, N. Dimarcq, and V. Giordano, "Influence of low magnetic field on pumping efficiency in an optically pumped cesium beam resonator," in Proc. Frequency Standards and Metrology, A. DeMarchi, Ed. New York: Springer-Verlag 1988, pp. $110-115$. 